\title{
lleo-colic intra-corporeal anastomosis during robotic right colectomy: a systematic literature review and meta-analysis of different techniques
}

\author{
Simone Guadagni ${ }^{1} \cdot$ Matteo Palmeri $^{1} \cdot$ Matteo Bianchini ${ }^{1}$ - Desirée Gianardi ${ }^{1} \cdot$ Niccolò Furbetta $^{1} \cdot$ Fabrizio Minichilli $^{2}$. \\ Gregorio Di Franco $^{1} \cdot$ Annalisa Comandatore ${ }^{1} \cdot$ Giulio Di Candio ${ }^{1} \cdot$ Luca Morelli $^{1,3}$ (i)
}

Accepted: 15 January 2021 / Published online: 23 January 2021

(C) The Author(s) 2021

\begin{abstract}
Purpose Robotic assistance could increase the rate of ileo-colic intra-corporeal anastomosis (ICA) during robotic right colectomy (RRC). However, although robotic ICA can be accomplished with several different technical variants, it is not clear whether some of these technical details should be preferred. An evaluation of the possible advantage of one respect to another would be useful. Methods We conducted a systematic review of literature on technical details of robotic ileo-colic ICA, from which we performed a meta-analysis of clinical outcomes. The extracted data allowed a comparative analysis regarding the outcome of overall complication (OC), bleeding rate (BR) and leakage rate (LR), between (1) mechanical anastomosis with robotic stapler, versus laparoscopic stapler, versus totally hand-sewn anastomosis and (2) closure of enterocolotomy with manual double layer, versus single layer, versus stapled.

Results A total of 30 studies including 2066 patients were selected. Globally, the side-to-side, isoperistaltic anastomosis, realized with laparoscopic staplers, and double-layer closure for enterocolotomy, is the most common technique used. According to the meta-analysis, the use of robotic stapler was significantly associated with a reduction of the BR with respect to mechanical anastomosis with laparoscopic stapler or totally hand-sewn anastomosis. None of the other technical aspects significantly influenced the outcomes.

Conclusions ICA fashioning during RRC can be accomplished with several technical variants without evidence of a clear superiority of anyone of these techniques. Although the use of robotic staplers could be associated with some benefits, further studies are necessary to draw conclusions.
\end{abstract}

Keywords Robotic surgery $\cdot$ Intra-corporeal anastomosis $\cdot$ Right colectomy $\cdot$ da Vinci

\section{Introduction}

The application of minimally invasive surgery in colorectal procedures has rapidly spread worldwide. In particular,

Luca Morelli

luca.morelli@unipi.it

1 General Surgery Unit, Department of Translational Research and new Technologies in Medicine and Surgery, University of Pisa, Via Paradisa 2, 56124 Pisa, Italy

2 Unit of Environmental Epidemiology and Disease Registries, Institute of Clinical Physiology, National Council of Research, Pisa, Italy

3 Endo-CAS (Center for Computer Assisted Surgery), University of Pisa, Pisa, Italy laparoscopic right colectomy is associated with earlier return to normal bowel function, shorter length of hospital stay, fewer wound complications and similar oncological outcomes respect to the conventional open approach [1]. Thus, minimally invasive right colectomy is nowadays a commonly performed procedure.

However, in spite of the well-demonstrated benefits of the complete intra-corporeal anastomosis (ICA), such as the faster bowel recovery and less analgesic usage, thanks to the reduced mesenteric traction, with respect to the extracorporeal technique [2, 3], its widespread usage during a minimally invasive right colectomy is still limited.

Indeed, due to the laparoscopic skills required, especially in terms of suturing, it is estimated that less than $10 \%$ of procedures are accomplished with the fashioning of ileo-colic ICA 
[4] and most surgeons performing laparoscopic right colectomy still use the extracorporeal approach.

In this context, the application of robotic surgery has been considered an appealing advancement. The da Vinci robotic system has gained popularity in colorectal surgery because it has been expected to overcome the steering learning curve of laparoscopy and to allow an easier access to narrow spaces. A lot of papers have shown a reduction of conversion rates also in 'difficult cases' [5]. Moreover, thanks to the introduction of the da Vinci Xi platform, together with its specific tools, the two main drawbacks of robotic surgery, operative time and costs, seem to be flattened [6, 7]. Recently, the attention has been focused on right colectomy with the introduction of robotic 'top to down' complete mesocolic excision and the sovra-pubic approach $[8,9]$. In all these surgical procedures, robotic assistance may decrease workload and improve suturing performance. Therefore, by overcoming the kinematic limitations of pure laparoscopy, it could play a key role also in increasing the adoption rate of ICA during robotic right colectomy (RRC).

Similarly to laparoscopy, robotic ICA can be accomplished with several different technical variants, such as, for instance, mechanical or totally hand-sewn, single- or double-layered, and by using several types of suture. As until now it is not clear if some of these technical details should be preferred, we aimed to provide a systematic review of the literature about ICA during RRC and to compare the different anastomosis variants evaluating intra- and post-operative outcomes, in order to find if one of these surgical techniques may be superior to the others.

\section{Methods}

An extensive literature review from inception to March 2020 using PubMed database for English literature was performed. Research question was are there any differences in terms of clinical outcomes between the several technical variants of robotic intracorporeal ileo-colic anastomosis fashioning? The searched formulas were 'Robotic AND intra-corporeal anastomosis', 'Robotic AND ileo-colic anastomosis', 'Robotic AND right colectomy', 'Robotic AND complete mesocolic excision', 'Robotic AND transverse colon'.

Article selection was carried out according to the preferred reporting items for systematic reviews and meta-analyses criteria (PRISMA) [10] (Fig. 1) and AMSTAR (assessing the methodological quality of systematic reviews) guidelines. Manuscripts identified by cross-referencing were also retrieved and evaluated. Inclusion criteria were as follows: articles in English, reporting more than 10 robot-assisted right colectomies with ileo-colic ICA and in which the authors provided a detailed description of their robotic technique. Exclusion criteria were as follows: original articles with less than 10 patients, case reports, letters to the editor, editorial comments or other works without clinical records or without technical description of the anastomosis.

From articles, comparing robotic extra- and ICA, we extracted only technical and clinical data of the ICA group. In the same manner, from studies comparing laparoscopic and RRC, we extracted only technical and clinical data of the robotic group.

We extracted from each study the following data: number of patients, mean age, male/female ratio, type of anastomosis, stapler used, opening and closure of enterotomies. The suture type, the stapler and the cartridge adopted were also retrieved. Finally, the complication rate was recorded with attention to overall complications (OC), anastomotic leakage and bleeding. OC was defined as overall post-operative complications, both medical and surgical following the Clavien-Dindo classification [11], and reported by the authors. Leakage rate (LR) was defined as any enteral leak from ICA that was treated conservatively or that required any interventional procedure in the post-operative course, and reported by the authors. Bleeding rate (BR) was defined as any intraluminal haemorrhage from the anastomotic site that was treated conservatively or that required any interventional procedure in the postoperative course, and reported by the authors.

Other details not specifically inherent to the ICA technique, such as pre-operative diagnosis, histopathologic diagnosis, number of lymph nodes harvested, follow-up beyond the post-operative course, were not extracted in the present review because not useful for the purpose of this study.

From the systematic review, we also performed a metaanalysis of clinical outcomes, including all patients of those articles for which it was possible to obtain adequate data for a statistically significant analysis, and for which all the details were clearly deducible.

Three authors (SG, MB and NF) independently reviewed all the manuscripts that met the inclusion criteria. The final search was completed by April 15, 2020. This systematic review and meta-analysis has been registered on PROSPERO [registration number CRD42020213777].

\section{Statistical analysis}

Meta-analysis was performed using the 'metaprop' routine [12] by the Stata statistics software (version 15 for Windows Stata Corporation, 2017). The metaprop routine entails the Freeman-Tukey double arcsine transformation procedure and DerSimonian-Laird random-effects model [13, 14]. Specifically, the Freeman-Tukey double arcsine procedure transforms proportions from individual studies by stabilizing between-study variance. Subsequently, the DerSimonianLaird random-effects model computes the weighted overall pooled estimates. Between-study heterogeneity was assessed by inspecting the forest plots and the chi-squared test for 
Fig. 1 PRISMA diagram of literature research
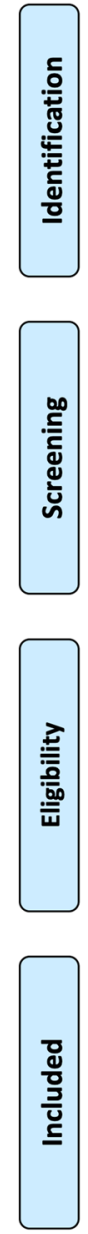

heterogeneity. $I^{2}$ statistic with a value above $50 \%$ was interpreted as representing high heterogeneity [15], and therefore, a random-effects model analysis was used. When heterogeneity was modest $\left(I^{2}<50 \%\right)$, a fixed-effects model of analysis was performed. Results of the meta-analysis were reported as pooled prevalence of OC, LR and BR with $95 \%$ confidence intervals (CIs); $p$ values $<0.05$ were considered statistically significant.

\section{Results}

\section{Descriptive analysis}

Using the search terms listed above, 413 publications were identified. No randomized trial was found. After title and abstract review, 353 articles were excluded as duplicate or nonpertinent. The remaining 60 studies were investigated in detail. Thirty of them were than excluded for the following reasons: articles without clinical records $(n=10)$, case reports $(n=14)$, case series with less than 10 patients involved $(n=$ $6)$. Then, we finally selected 30 studies $[8,15-43]$ involving a

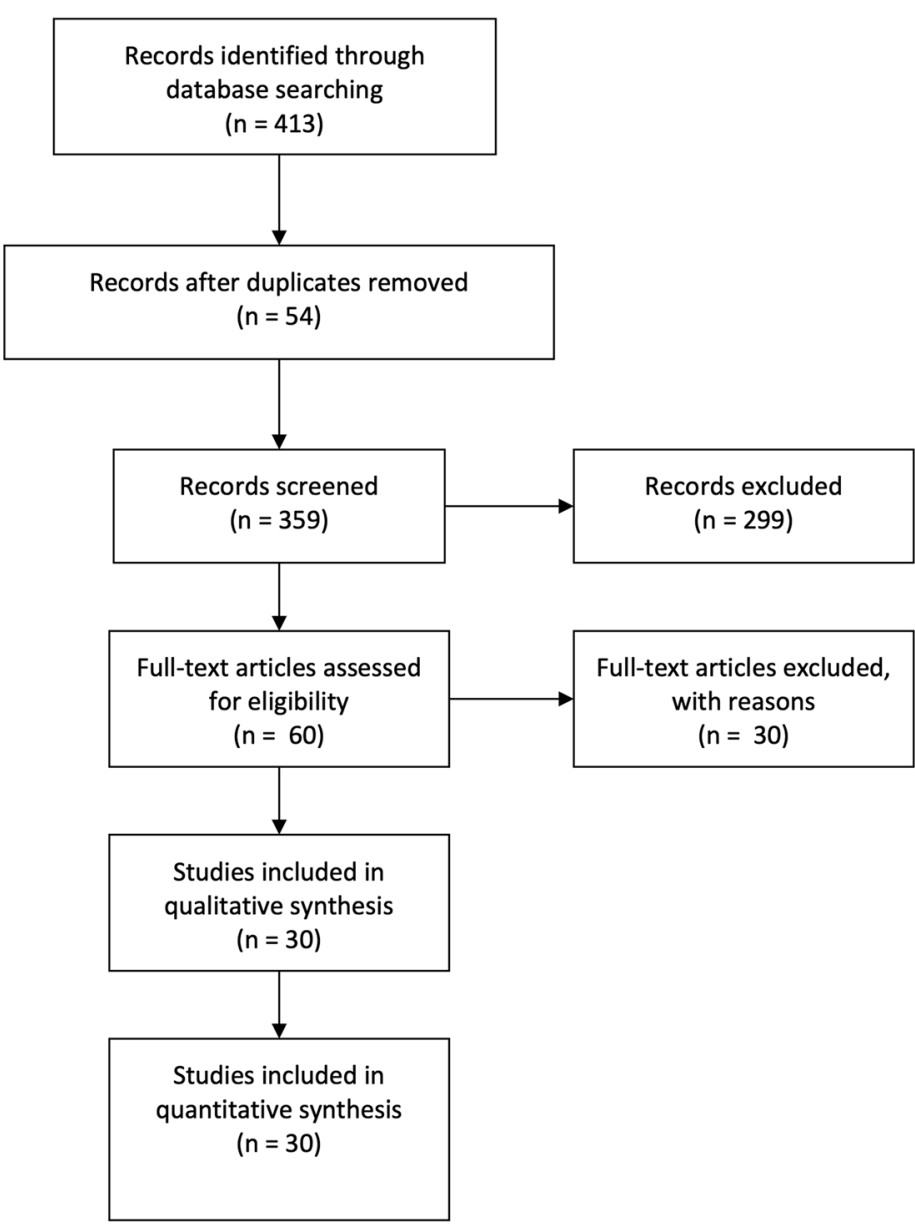

total of 2066 patients (Table 1). The only article that specifically compared different techniques used during minimally invasive ICA (either robotic and laparoscopic) was conducted by Milone et al. and was included in our review for the robotic part.

In eight manuscripts, the robotic platform used was the da Vinci Xi (Intuitive Surgical Inc., Sunnyvale, CA, USA); in six manuscripts, both the da Vinci Xi and the da Vinci Si were used, whereas in twelve manuscripts, the da Vinci Si was the only robotic system considered. In four papers, the specific robotic platform used was not mentioned.

Eight articles [8, 16-22] described the use of robotic stapler in fashioning an ileo-colic anastomosis. All the anastomoses were mechanical isoperistaltic. The type of cartridge was specified only in three articles (Kelly et al. [17], Blumberg et al. [20] and Johnson et al. [21]), with the blue load used for all of them. The main difference arisen from these studies concerns the closure of the enterocolotomy. Five studies described a double-layer closure: Scotton et al. [16] used a 3/0 Assufil, Kelly et al. [17] a 3/0 Quill suture, whereas Hamzaoglu et al. [8] used 3/0 V-Loc suture. Ozben et al. [19] closed the enterocolotomy in a double layer but they 


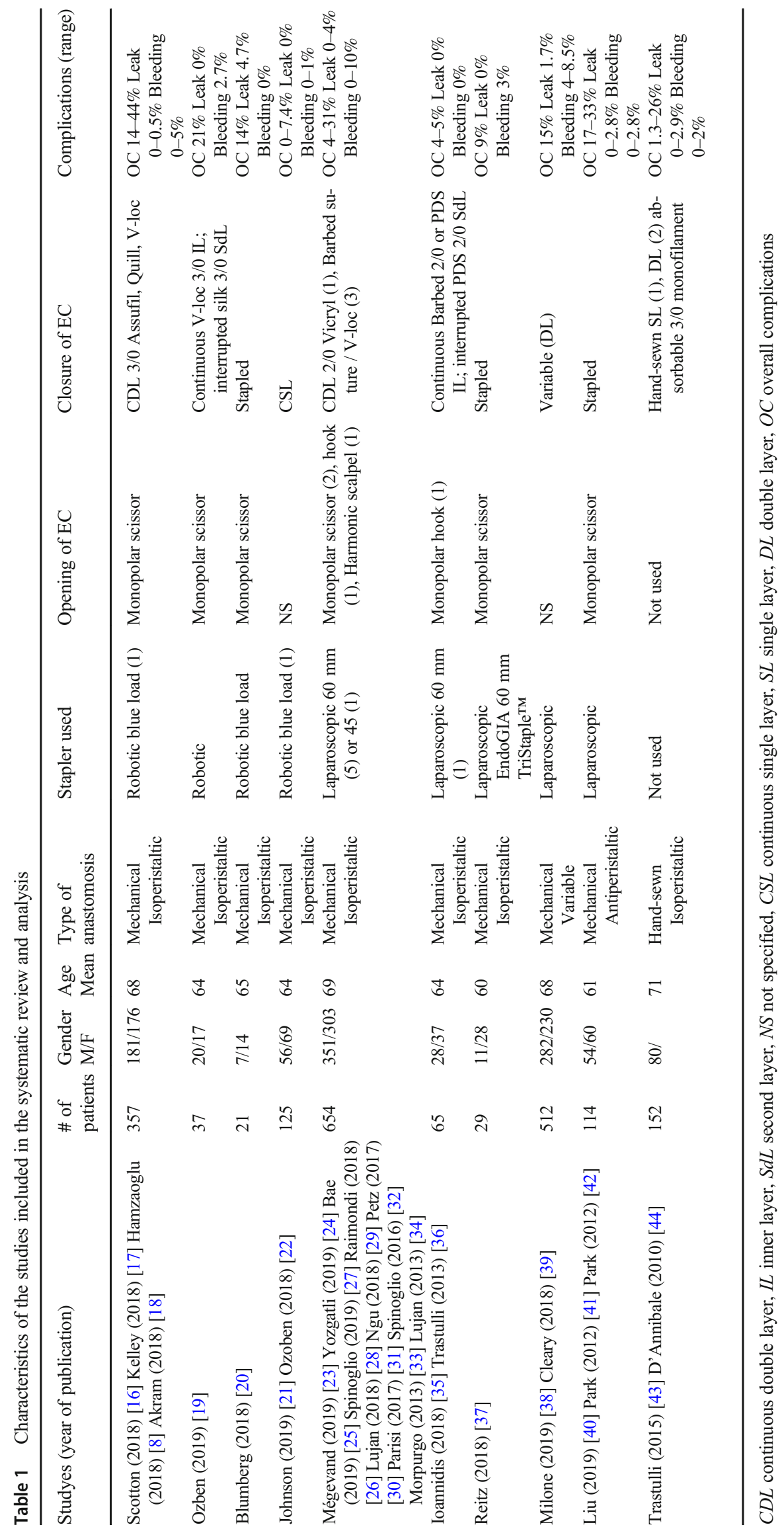


preferred continuous 3/0 V-Loc for the inner layer and interrupted 3/0 silk for the second one. Instead, two manuscripts reported a single layer continuous suture in this phase, without specifying the type of suture [21, 22]. Only Blumberg et al. [20] in 2018 closed the defect with another firing of robotic $3.5 \mathrm{~mm}$ stapler.

The use of a laparoscopic stapler was reported in twenty articles [23-42] with only two manuscripts (Bae et al. [25] and Petz et al. [30]) describing the use of both robotic and laparoscopic staplers.

The mechanical isoperistaltic orientation for ICA fashioning was reported in fifteen articles [23-37] and it was the most used one in the two multi-center trials (performed by Milone et al. [38] and Cleary et al. [39]). Anti-peristaltic orientation was reported only by three articles [40-42].

Considering the type of laparoscopic stapler, in two articles, a $45 \mathrm{~mm}$ Echelon Endopath was used; in five articles, the mechanical anastomosis was performed with $60 \mathrm{~mm}$ Echelon Endopath (with the blue load described in two articles). Only in one case series, the anastomosis was created with EndoGIA Tri-Stapler purple load. In the remaining studies, the type of laparoscopic stapler used was not mentioned. The closure of enterocolotomy was performed with double layer 2/0 Vicryl by Lujan et al. [28], while double layer with barbed/self-anchoring sutures was described by Yozgatli et al. [24], Bae et al. [25] and Raimondi et al. [26] respectively. Ioannidis et al. [35] used a continuous barbed suture for the inner layer and an interrupted 2/0 PDS for the second layer, while Trastulli et al. [36] used a continuous 2/0 PDS suture for the inner layer and an interrupted 2/0 PDS for the second layer. In the remaining eight studies, the type of suture used for doublelayer closure of enterocolotomy was not mentioned.

Only in two manuscripts, a totally hand-sewn ICA after right colectomy was considered by Trastulli et al. [43] and D'Annibale et al. [44]. The first authors used a single layer of 3/0 absorbable monofilament suture; meanwhile, D'Annibale et al. performed it with a double layer of 3-0 absorbable monofilament.

The extracted data allowed a statistically significant comparative analysis regarding the outcome of $\mathrm{OC}$, bleeding rate (BR) and leakage rate (LR), between (1) mechanical anastomosis with robotic stapler, versus laparoscopic stapler, versus totally hand-sewn anastomosis and (2) closure of enterocolotomy with manual double layer, versus manual single layer, versus stapled.

It was not possible to compare the type of suture used or sutures' characteristics (such as running or interrupted sutures), due to the small number and the high heterogeneity of the reported data on articles that specifically described these aspects. For the same reasons, differences in cartridge load during robotic or laparoscopic mechanical anastomosis were not statistically analysed.

\section{Meta-analysis}

\section{Mechanical anastomosis with robotic stapler, versus laparoscopic stapler, versus totally hand-sewn anastomosis}

A total of thirty articles comprising 2066 patients were considered. The mechanical anastomosis with robotic stapler group (MC Rob group) included eight studies with a total of 540 patients involved, whereas the mechanical anastomosis with laparoscopic stapler group (MC Lap group) included twenty studies with a total of 1374 patients involved. Finally, totally hand-sewn anastomosis group (TS group) included two studies with a total of 152 patients. Leakage and bleeding rates were retrieved from all articles, while $\mathrm{OC}$ was retrieved from twenty-seven manuscripts ( $96 \%$ of total).

\section{Pooled prevalence of $\mathrm{OC}$}

The estimated overall pooled prevalence of OC was $16.45 \%$ (95\% CI 11.83-21.61). The pooled prevalence of OC for the MC Rob group was $16.20 \%$ (95\% CI .01-33.42), the pooled prevalence of OC among patients in MC Lap group was $16.89 \%$ (95\% CI 12.92-21.23), while the prevalence among patients in TS group was $16.12 \%$ (95\% CI 10.59-22.51). Studies conducted in MC Rob group had a considerably higher heterogeneity $\left(I^{2}=94.12 \%\right)$ than those in Lap group $\left(I^{2}=65.18 \%\right)$. The difference between the three groups was not statistically significant ( $p=0.949)$ (Fig. 2).

\section{Pooled prevalence of LR}

Considering all groups, no heterogeneity between studies was detected. The fixed estimate overall pooled prevalence of leakage was $0.09 \%$ (95\% CI 0-0.42). The estimated pooled prevalence of leakage for MC Rob and MC Lap groups was $0 \%$ (95\% CI 0-0.18) and 0.18\% (95\% CI 0-0.68) respectively, while in TS group, the prevalence was $1.50 \%$ (95\% CI 0.02 4.39). The difference between all groups was not statistically significant ( $p=0.273)$ (Fig. 3).

\section{Pooled prevalence of BR}

The overall fixed-effects pooled prevalence of bleeding was $1.12 \%$ (95\% CIs: $0.59-1.78$ ) with a low heterogeneity between studies $\left(I^{2}=34.52 \%\right)$. When only MC Lap group was considered, the pooled prevalence increased to $1.83 \%$ (95\% CIs: 1.04-2.78; heterogeneity $I^{2}=35.83 \%$ ). Considering both MC Rob group and TS groups, no heterogeneity between studies resulted $\left(I^{2}=0 \%\right)$. The fixed-effects pooled prevalence of MC Rob group were $0.04 \%$ (95\% CIs: $0-0.76$ ). For TS group, the pooled prevalence was $0.98 \%$ (95\% CIs: 0-3.56). The difference between groups was statistically significant 


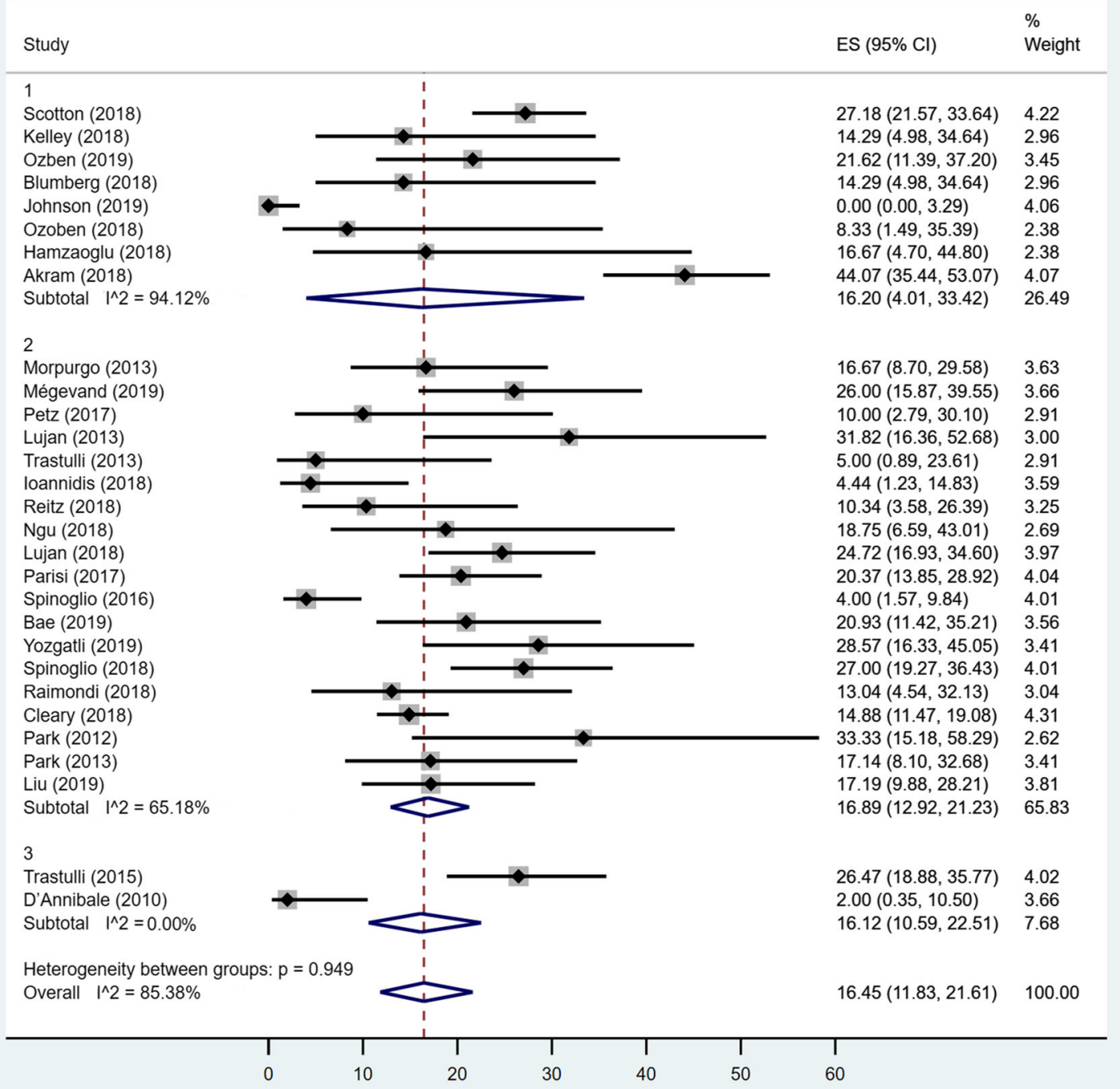

Fig. 2 Forest plot showing OC in MC Rob group (1), MC Lap group (2), TS group (3) and overall studies

( $p=0.026)$ with a lower BR in the MC Rob group (0.04\%) with respect to MC Lap group (1.83\%) and to TS group (0.98\%) (Fig. 4).

\section{Closure of enterocolotomy with double layer, versus single layer, versus stapled}

A total of twenty-six articles with 1402 patients were considered. We excluded from this analysis the studies conducted by Milone et al. and Cleary et al., as we cannot define exactly the total number of patients in which the closure of enterocolotomy during robotic mechanical ICA was performed in a single or double layer. The first group, mechanical ICA with double-layer closure of enterocolotomy (MD group), comprised nineteen articles with a total of 1113 patients; the second group, mechanical ICA with single layer closure of enterocolotomy (MS group), included two papers with a total of 125 patients. The third group, mechanical ICA with stapled closure of enterocolotomy (MSt group), included five articles with a total of 164 patients.

\section{Pooled prevalence of $O C$}

The overall random-effects pooled prevalence of OC was $16.95 \%$ (95\% CIs: 11.64-22.95) with a high level of heterogeneity $\left(I^{2}=85.29 \%\right)$ (Fig. 5). When only studies concerning MD group were considered, the pooled prevalence increased to $19.28 \%$ (95\% CIs: 13.97-25.16), with reduced, but still high, heterogeneity $\left(I^{2}=78.81 \%\right)$. For both MSt and MS groups, the $I^{2}$ was $0 \%$; the MSt pooled prevalence of OC was $16.51 \%$ (95\% CIs: 10.92-22.88) and for MS group, the 


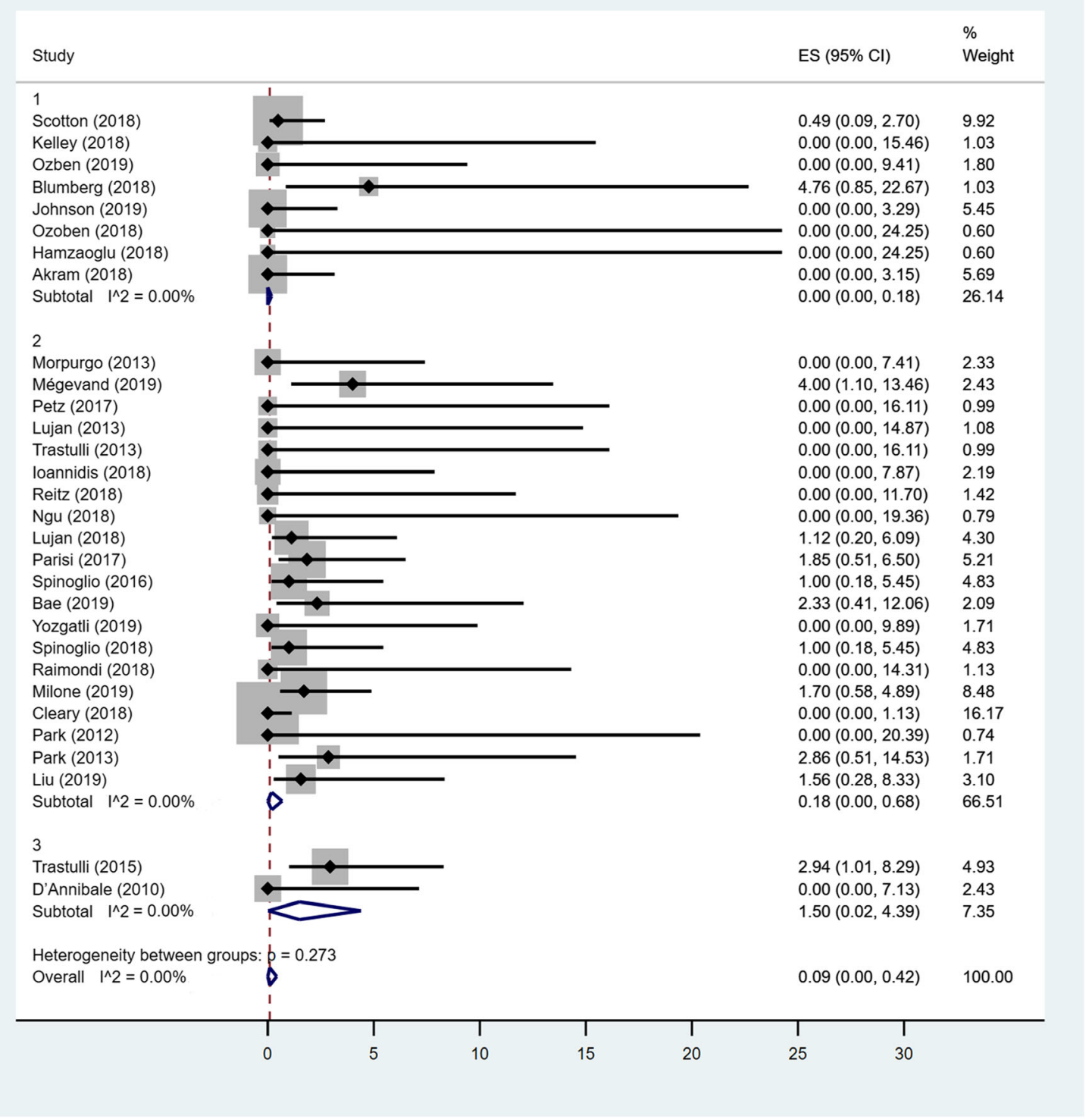

Fig. 3 Forest plot showing leakage rate in MC Rob group (1), MC Lap group (2), TS group (3) and overall studies

pooled prevalence was $0 \%$ (95\% CIs: $0-0.93$ ). The difference between groups was statistically significant $(p<0.001)$ with a higher OC in the MD group (19.28\%) and MSt group (16.51\%) with respect to MS group (0\%) (Fig. 5).

\section{Pooled prevalence of LR}

Considering that in all groups, there was no heterogeneity between studies $\left(I^{2}=0\right)$; then, the fixed effects model was used to estimate the pooled prevalence. The overall pooled prevalence of leakage was $0.23 \%$ (95\% CI $0-0.72$ ). The pooled prevalence of leakage for the MD group was $0.17 \%$ (95\% CI 0-0.72). The pooled prevalence of leakage for the MSt group was $1.24 \%$ (95\% CI 0.11-3.11), while the prevalence among patients in MS groups was $0 \%$ (95\% CI 0-0.45).
The difference between groups was not statistically significant $(p=0.152)$ (Fig. 6).

\section{Pooled prevalence of BR}

Considering that in all groups, there was no heterogeneity between studies $\left(I^{2}=0\right)$; then, the fixed effects model was used to estimate the pooled prevalence. The overall pooled prevalence of bleeding was $0.39 \%$ (95\% CI $0-1)$. The pooled prevalence for the MS group was $0.53 \%$ (95\% CI 0-3.56), while the pooled prevalence among patients in MD and MSt groups was $0.39 \%(95 \% \mathrm{CI} 0.02-1.08)$ and $0.39 \%(95 \% \mathrm{CI}$ $0-2.71$ ), respectively. The difference between groups was not statistically significant ( $p=0.861)$ (Fig. 7). 


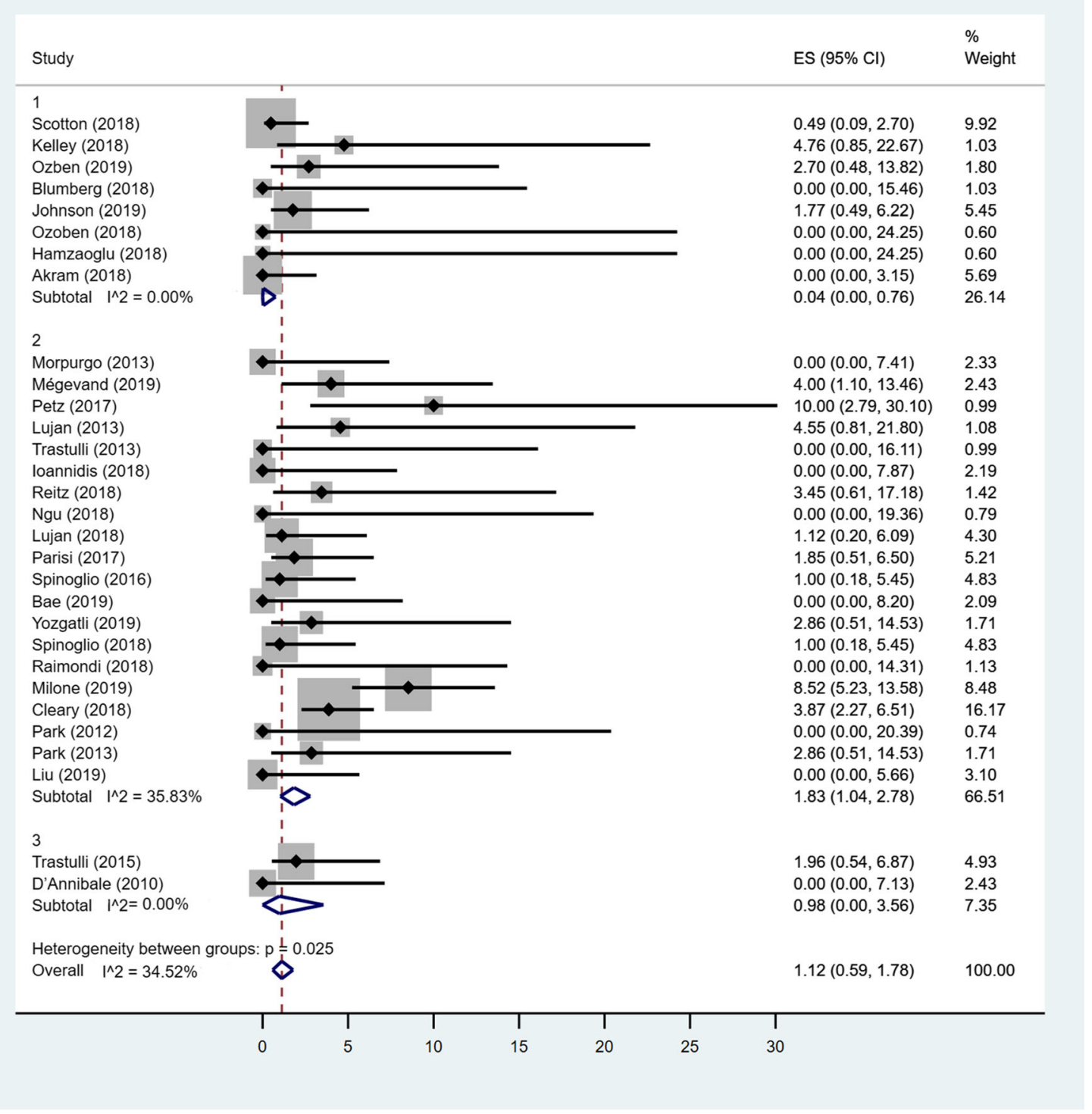

Fig. 4 Forest plot showing bleeding rate in MC Rob group (1), MC Lap group (2), TS group (3) and overall studies

\section{Discussion}

From the early nineties, conventional direct manual laparoscopy had a rapid growth in several surgical scenarios, and nowadays, it is considered a cornerstone in colorectal surgery [45]. In particular, laparoscopic right colectomy has modified some traditional acquisitions; for example, it has introduced the medio-lateral dissection and the possibility to perform an extracorporeal anastomosis or an intra-corporeal one [3]. Although several studies have underlined some advantages of ICA over extracorporeal anastomosis [46, 47], it inevitably requires dexterity and more advanced laparoscopic skills, thus limiting its worldwide diffusion. In fact, in most cases, only expert laparoscopic surgeons are faced with such a technique [48]. The robotic approach, with its technological advantages, makes some surgical manoeuvres easier. In particular, thanks to these aspects and to the introduction of robotic staplers, there is a growing interest and expansion of robotic assistance during right colectomy, due to the facilitating effect that could have an impact in the reconstructive phase, contributing to the greater adoption of ICA also from less experienced surgeons. However, in spite of the increasing number of robotic systems installed, and of the increasing number of robotic right colectomies with ICA performed, it is still not sufficiently studied whether some technical details in performing the reconstruction phase robotically are superior to others.

The present review focuses on robotic ICA during right colectomy and summarises the evidence about the common techniques used. Globally, from the literature review, we found that the side-to-side, isoperistaltic anastomosis, realized with laparoscopic staplers and double-layer closure for enterocolotomy, is the most common technique used so far. 


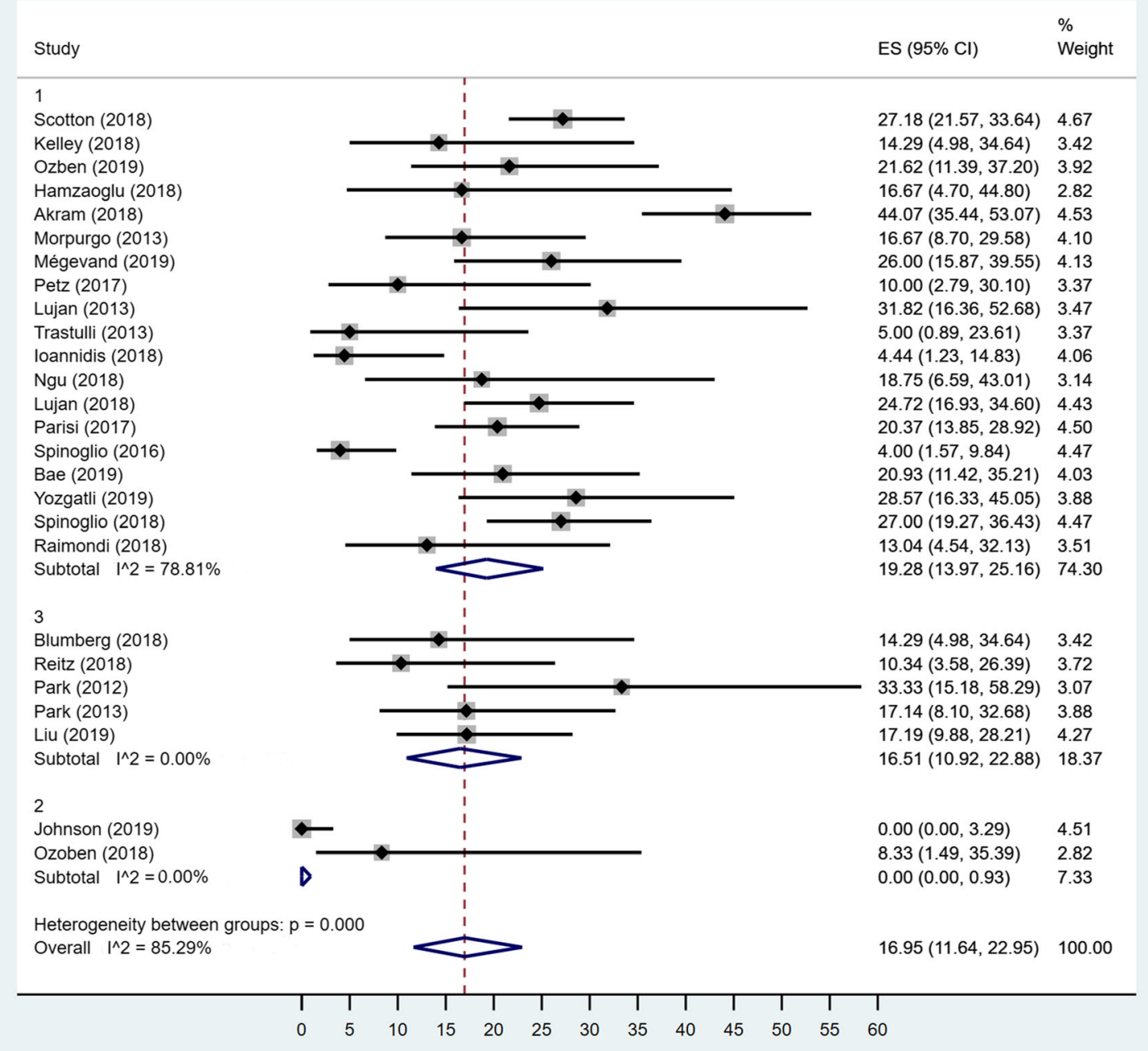

Fig. 5 Forest plot showing OC in MD group (1), MS group (2), MSt group (3) and overall studies

However, several other variants, such as the use of robotic staplers, the totally hand-sewn anastomosis, the closure of enterocolotomy in single layer with different kind of sutures or stapled, have been significantly reported as well. Proceeding with our meta-analysis from the articles included in the review, we were able to obtain data statistically analysable within two different major groups. Thus, regarding the type of anastomosis, only for the use of robotic stapler, versus laparoscopic stapler, versus totally hand-sewn anastomosis, it was possible to obtain data to be analysed. Similarly, regarding the type of enterocolotomy closure, we could compare only the results of double layer, versus single layer, versus stapled. With respect to other further details, the heterogeneity of the studies and/or the lack of information made it possible to perform only a qualitative description.

Concerning the type of anastomosis, from the meta-analysis, we observed that the OC prevalence comparing the mechanical anastomosis with robotic and laparoscopic staplers or totally sewn, is in line with current literature regarding $\mathrm{OC}$ in the classic laparoscopic right colectomies [49], without obtaining though any significant difference between the three subgroups. This confirms the safety of the robotic procedure, which is comparable to the widely used laparoscopic technique, giving us also the indication that none of these different choices can be considered superior so far, with respect to the others. Also, when considering LR, we did not find any difference between the three different subgroups. However, when dealing with BR, we found in MC Rob group a reduction of BR rate respect to the others two subgroups. This may be related to the technical aspects intrinsic to robotic staplers, such as the smart clamp ${ }^{\circledR}$ technology which partially restores the presence of some 'intelligent feedback', by measuring the jaw closure and displaying objective feedback before firing, to optimize staple line formation. However, as far as we know, no articles in literature exist about this specific topic, and therefore, further studies are needed to investigate it and to draw conclusions. 


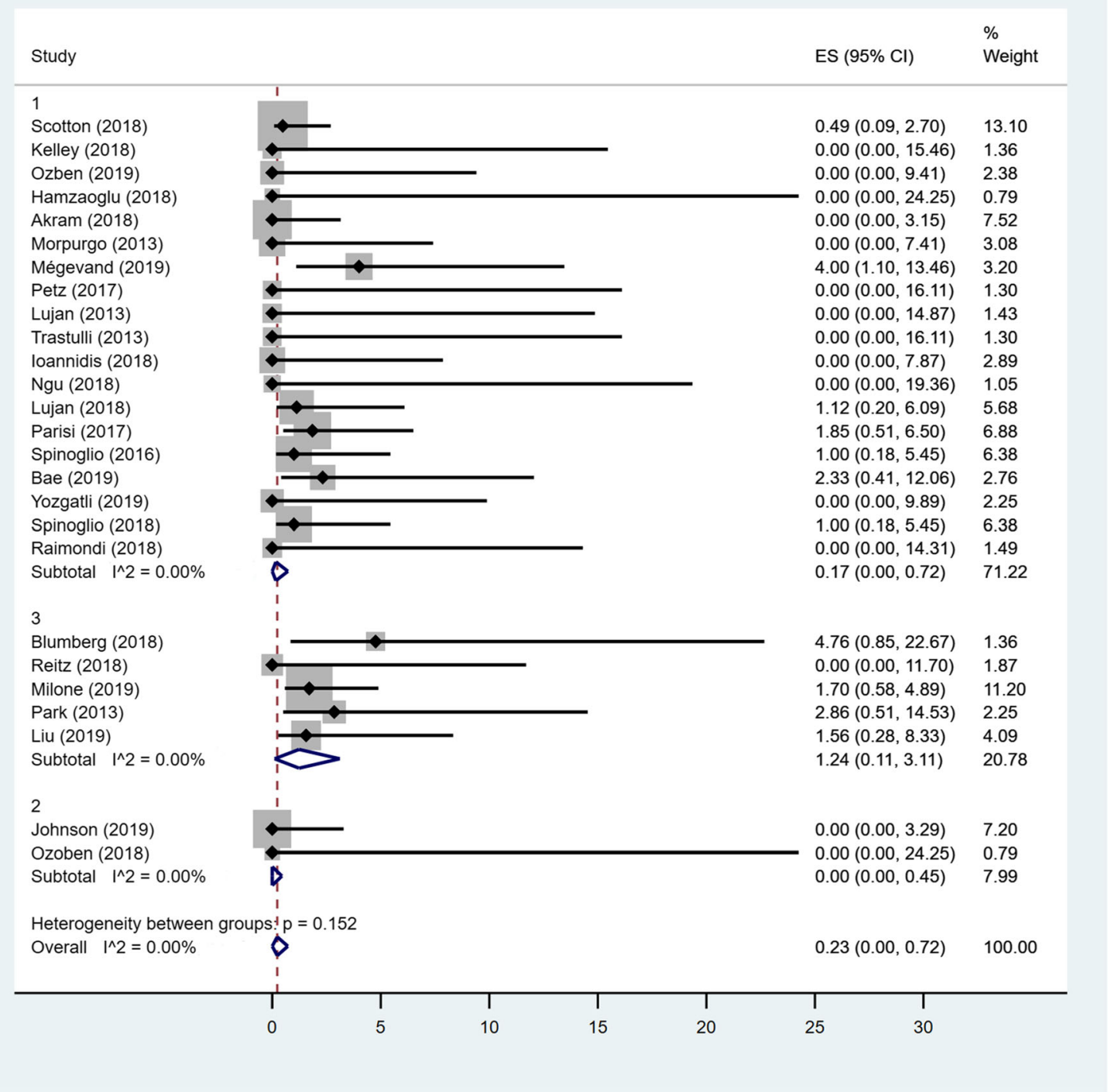

Fig. 6 Forest plot showing leakage rate in MD group (1), MS group (2), MSt group (3) and overall studies

Considering the closure of entero-colotomy, most of the works in literature report a double-layer technique, by using different sutures such as Assufil, Quill suture, Vicryl or barbed suture like V-Loc.

In apparent contrast with data from laparoscopic experience [38], the leakage and bleeding rates related to the closure of entero-colotomy in a single layer during robotic approach are not significantly higher respect to the double-layer closure. These good results of robot-assisted single layer closure respect to laparoscopy might come from the defined advantages of robotic Endo-Wrist instruments, 3D vision and suitable operative field, contributing to the general good quality of robotic suture. However, it is also possible that these similar results between SL and DL closure in robotic series are still affected by the relatively small number of procedures respect to the laparoscopic ones, and in future the superiority of DL could be assessed also in robotic procedures, with the availability of more data. Considering OC in these three subgroups, the results are again in line with current literature regarding $\mathrm{OC}$ in the classic laparoscopic right colectomies [49], although we noted a significant difference between the three study groups, with a lower rate of OC in MS group. However, these data seem to be not very meaningful due to the high level of heterogeneity and particularly to the great difference between the too little data on the MS group compared with the very high number of studies and therefore reported patients in the remaining two groups. Furthermore, as OC are related to several factors that may have affected the results, often beyond the mere surgical technical aspects, we think that these results should be considered a consequence of a bias. On contrary, surgical complications and in particular $\mathrm{BR}$ and LR are more related to intrinsic technical aspects, thereby reducing the possible impact of other variables when dealing with these aspects. In this regard, since a specific work 


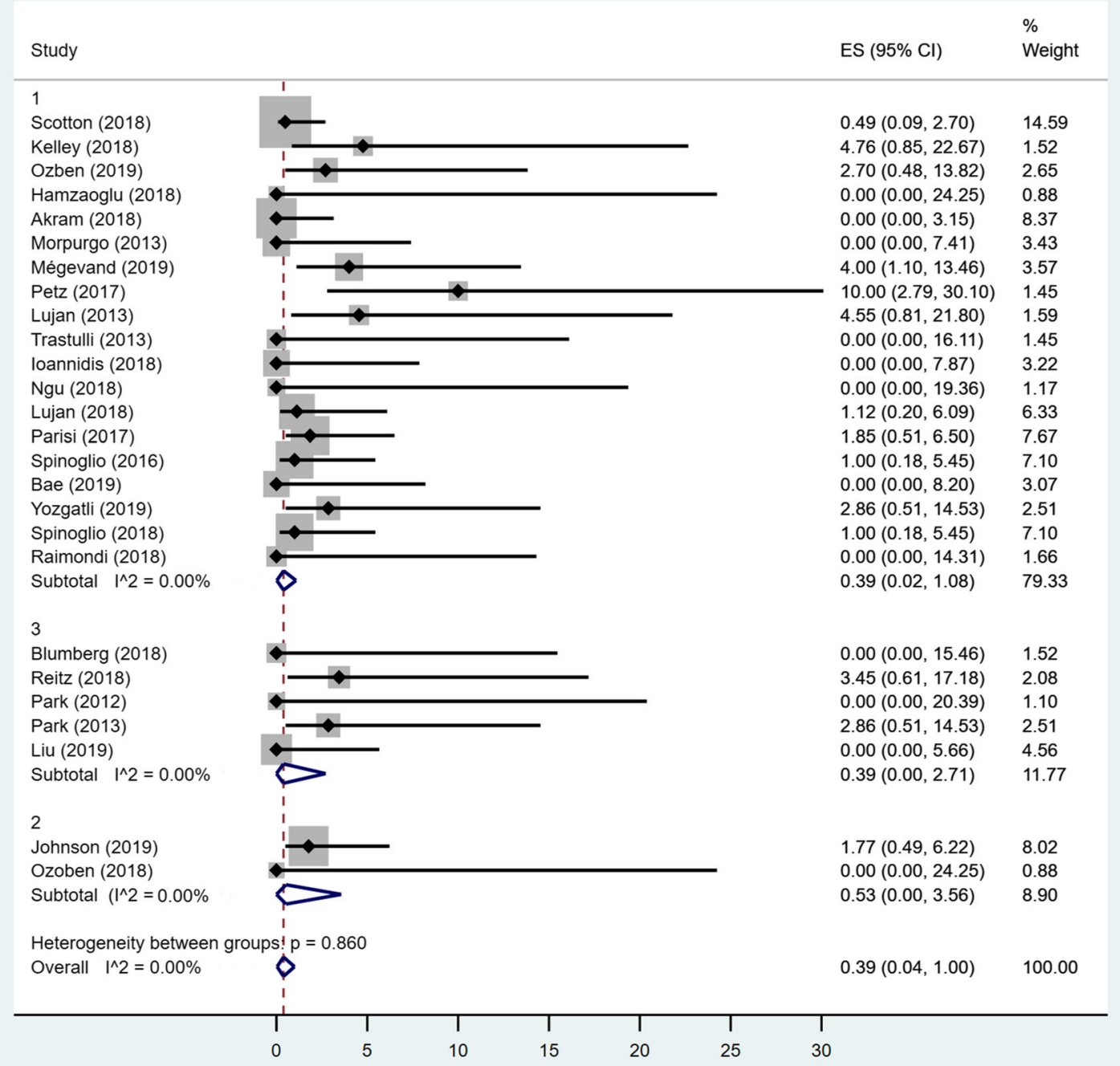

Fig. 7 Forest plot showing bleeding rate in MD group (1), MS group (2), MSt group (3) and overall studies

about the enterocolotomy closure during laparoscopic fashioning of ICA in right colectomy strongly defines the superiority of DL respect to SL [38], and since the robotic assistance allows to complete DL closure without any particular effort, we think that the most cautious procedure to be adopted during robotic ICA is still the DL respect to the SL, until further solid data will be available.

Finally, it would have been useful to analyse also data about the type of suture, but unfortunately, because of the heterogeneity of the articles on this aspect, it was not possible. Thus, being quite difficult to draw definitive conclusions, we can comment this aspect only qualitatively. The safety and efficacy of barbed sutures with regards of V-Loc were just provided in recent publications for laparoscopic ICA [50, 51]. These sutures seem to be suitable during robotic assistance in which the absence of tactile feedback may limit the sutures tighten. However, the only article that extensively evaluated different ways to close the entero-colotomy during mechanical ICA after right colectomy was conducted by Milone et al. [38]. They recommended a double-layer closure using a running barbed suture in the first one. Moreover, no differences in terms of operative time and complications were noted between laparoscopic and robotic ICA. However, this multi-institutional analysis included only highly experienced centers, and this may represent a drawback.

This review has some intrinsic limitations that should be pointed out. Firstly, although we used a I2 statistic method, the studies' heterogeneity, and the absence of all technical detailed information, limits our conclusions. Moreover, the difference in surgical robotic expertise among the included studies, as well as the absence of randomized trial, may also interfere with our results. However, to the best of our knowledge, the present article is the first to summarize different technical aspects about the use of robot during ICA in right colectomy 
and we found some interesting descriptive results, alongside the meta-analysis: according to available literature, the sideto-side isoperistaltic mechanical anastomosis and the doublelayer closure for enterocolotomy are the wider adopted techniques for ICA fashioning during right colectomy, with a trend toward a standardized technique in clinical practice.

\section{Conclusions}

Robotic ICA during right colectomy is a safe procedure. It can be accomplished according to several technical variants, either regarding the type of stapler used, the type of enterocolotomy closure technique, or it can be performed totally hand sewn. According to data from available literature, there is no evidence of a superiority of any of these surgical techniques respect to another. The side-to-side isoperistaltic anastomosis, realized with laparoscopic staplers and double-layer closure for enterocolotomy, is the most commonly used technique. There could be a reduction in bleeding rate with the use of robotic staplers, likely related to the technical innovations brought by the smart clamp® technology. Given the widespread of robotic surgery, and the possible impact it may have in the diffusion of ICA during right colectomy, further studies, hopefully randomized, are necessary to clarify whether a superiority may exist among one of these technical variants.

Funding Open Access funding provided by Università di Pisa. The study was supported by the ARPA foundation (www.fondazionearpa.it).

Data availability All data generated or analysed during this study are included in this published article (and its supplementary information files).

\section{Compliance with ethical standards}

Competing interests The authors declare no competing interests.

Consent to participate For this type of study, formal consent is not required.

Open Access This article is licensed under a Creative Commons Attribution 4.0 International License, which permits use, sharing, adaptation, distribution and reproduction in any medium or format, as long as you give appropriate credit to the original author(s) and the source, provide a link to the Creative Commons licence, and indicate if changes were made. The images or other third party material in this article are included in the article's Creative Commons licence, unless indicated otherwise in a credit line to the material. If material is not included in the article's Creative Commons licence and your intended use is not permitted by statutory regulation or exceeds the permitted use, you will need to obtain permission directly from the copyright holder. To view a copy of this licence, visit http://creativecommons.org/licenses/by/4.0/.

\section{References}

1. Van Bree SH, Vlug MS, Bemelman WA et al (2011 Sep) Faster recovery of gastrointestinal transit after laparoscopy and fast-track care in patients undergoing colonic surgery. Gastroenterology 141(3):872-880

2. Tarta C, Bishawi M, Bergamaschi R (2013 Oct) Intracorporeal ileocolic anastomosis: a review. Tech Coloproctol 17(5):479-485

3. Scatizzi M, Kroning KC, Borrelli A et al (2010 Dec) Extracorporeal versus intracorporeal anastomosis after laparoscopic right colectomy for cancer: a case-control study. World J Surg 34(12): 2902-2908

4. Solaini L, Bazzocchi F, Cavaliere D, Avanzolini A, Cucchetti A, Ercolani G (2018 Mar) Robotic versus laparoscopic right colectomy: an updated systematic review and meta-analysis. Surg Endosc 32(3):1104-1110

5. Morelli L, Guadagni S, Di Franco G et al (2016 Dec) Da Vinci single site $\odot$ surgical platform in clinical practice: a systematic review. Int J Med Robot 12(4):724-734

6. Morelli L, Di Franco G, Guadagni S et al (2018 Feb) Robot-assisted total mesorectal excision for rectal cancer: case-matched comparison of short-term surgical and functional outcomes between the da Vinci Xi and Si. Surg Endosc 32(2):589-600

7. Morelli L, Di Franco G, Lorenzoni V et al (2019) Structured cost analysis of robotic TME resection for rectal cancer: a comparison between the da Vinci Si and Xi in a single surgeon's experience. Surg Endosc 33:1858-1869

8. Hamzaoglu I, Ozben V, Sapci I, Aytac E, Aghayeva A, Bilgin IA, Bayraktar IE, Baca B, Karahasanoglu T (2018 Aug) “Top down notouch" technique in robotic complete mesocolic excision for extended right hemicolectomy with intracorporeal anastomosis. Tech Coloproctol 22(8):607-611

9. Schulte Am Esch J, Iosivan SI, Steinfurth F et al (2019 Jul 1) A standardized suprapubic bottom-to-up approach in robotic right colectomy: technical and oncological advances for complete mesocolic excision (CME). BMC Surg 19(1):72

10. Moher D, Liberati A, Tetzlaff J, Altman DG, The PRISMA Group (2009 Jul 21) Preferred reporting items for systematic reviews and meta-analyses: the PRISMA statement. PLoS Med 6(7):e1000097

11. Dindo D, Demartines N, Clavien P-A (2004) Classification of surgical complications: a new proposal with evaluation in a cohort of 6336 patients and results of a survey. Ann Surg 240:205-201

12. Nyaga VN, Arbyn M, Aerts M (2014 Nov 10) Metaprop: a Stata command to perform meta-analysis of binomial data. Arch Public Health 72(1):39

13. Miller J (1978) The inverse of the Freeman-Tukey double arcsine transformation. Am Stat:138-138

14. DerSimonian R, Nan L (1986 Sep) Meta-analysis in clinical trials. Control Clin Trials 7(3):177-188

15. Higgins JP, Thompson SG (2002 Jun 15) Thompson. Quantifying heterogeneity in a meta-analysis. Stat Med 21(11):1539-1558

16. Scotton G, Contardo T, Zerbinati A et al (2018 Oct) From laparoscopic right colectomy with extracorporeal anastomosis to robotassisted intracorporeal anastomosis to totally robotic right colectomy for cancer: the evolution of robotic multiquadrant abdominal surgery. J Laparoendosc Adv Surg Tech A 28(10):12161222

17. Kelley SR, Duchalais E, Larson DW (2018 Nov 1) Short-term outcomes with robotic right colectomy. Am Surg 84(11):17681773

18. Akram WM, Al-Natour RH, Albright J et al (2018 Dec) A propensity score-matched comparison of intracorporeal and extracorporeal techniques for robotic-assisted right colectomy in an Enhanced Recovery Pathway. Am J Surg 216(6):1095-1100 
19. Ozben V, Aytac E, Atasoy D, Erenler Bayraktar I, Bayraktar O, Sapci I, Baca B, Karahasanoglu T, Hamzaoglu I (2019 Feb) Totally robotic complete mesocolic excision for right-sided colon cancer. J Robot Surg 13(1):107-114

20. Blumberg D (2019 Aug) Robotic colectomy with intracorporeal anastomosis is feasible with no operative conversions during the learning curve for an experienced laparoscopic surgeon developing a robotics program. J Robot Surg 13(4):545-555

21. Johnson CS, Kassir A, Marx DS, Soliman MK (2019 Feb) Performance of da Vinci Stapler during robotic-assisted right colectomy with intracorporeal anastomosis. J Robot Surg 13(1): $115-119$

22. Ozben V, de Muijnck C, Esen E, Aytac E, Baca B, Karahasanoglu T, Hamzaoglu I (2018 Dec) Is robotic complete mesocolic excision feasible for transverse colon cancer? J Laparoendosc Adv Surg Tech A 28(12):1443-1450

23. Mégevand JL, Amboldi M, Lillo E, Lenisa L, Ganio E, Ambrosi A, Rusconi A (2019 Mar) Right colectomy: consecutive 100 patients treated with laparoscopic and robotic technique for malignancy. Cumulative experience in a single centre. Updat Surg 71(1):151156

24. Yozgatli TK, Aytac E, Ozben V et al (2019 Feb) Robotic complete mesocolic excision versus conventional laparoscopic hemicolectomy for right-sided colon cancer. J Laparoendosc Adv Surg Tech A 26

25. Bae SU, Yang SY, Min BS (2019 Mar) Totally robotic modified complete mesocolic excision and central vascular ligation for rightsided colon cancer: technical feasibility and mid-term oncologic outcomes. Int J Color Dis 34(3):471-479

26. Spinoglio G, Bianchi PP, Marano A, Priora F, Lenti LM, Ravazzoni F, Petz W, Borin S, Ribero D, Formisano G, Bertani E (2019 Dec) Robotic versus laparoscopic right colectomy with complete mesocolic excision for the treatment of colon cancer: perioperative outcomes and 5-year survival in a consecutive series of 202 patients. Ann Surg Oncol 26(Suppl 3):884

27. Raimondi P, Marchegiani F, Cieri M, Cichella A, Cotellese R, Innocenti P (2018 Mar) Is right colectomy a complete learning procedure for a robotic surgical program? J Robot Surg 12(1): $147-155$

28. Lujan HJ, Plasencia G, Rivera BX, Molano A, Fagenson A, Jane LA, Holguin D (2018 Feb) Advantages of robotic right colectomy with Intracorporeal anastomosis. Surg Laparosc Endosc Percutan Tech 28(1):36-41

29. Ngu JC, Ng YY (2018 Dec) Robotics confers an advantage in right hemicolectomy with intracorporeal anastomosis when matched against conventional laparoscopy. J Robot Surg 12(4):647-653

30. Petz W, Ribero D, Bertani E, Borin S, Formisano G, Esposito S, Spinoglio G, Bianchi PP (2017 Nov) Suprapubic approach for robotic complete mesocolic excision in right colectomy: oncologic safety and short-term outcomes of an original technique. Eur $\mathrm{J}$ Surg Oncol 43(11):2060-2066

31. Parisi A, Scrucca L, Desiderio J, Gemini A, Guarino S, Ricci F, Cirocchi R, Palazzini G, D'Andrea V, Minelli L, Trastulli S (2017 Mar) Robotic right hemicolectomy: analysis of 108 consecutive procedures and multidimensional assessment of the learning curve. Surg Oncol 26(1):28-36

32. Spinoglio G, Marano A, Bianchi PP, Priora F, Lenti LM, Ravazzoni F, Formisano G (2016 Dec) Robotic right colectomy with modified complete mesocolic excision: long-term oncologic outcomes. Ann Surg Oncol 23(Suppl 5):684-691

33. Morpurgo E, Contardo T, Molaro R, Zerbinati A, Orsini C, D'Annibale A (2013 May) Robotic-assisted intracorporeal anastomosis versus extracorporeal anastomosis in laparoscopic right hemicolectomy for cancer: a case control study. J Laparoendosc Adv Surg Tech A 23(5):414-417
34. Lujan HJ, Maciel VH, Romero R, Plasencia G (2013 Jun) Laparoscopic versus robotic right colectomy: a single surgeon's experience. J Robot Surg 7(2):95-102

35. Ioannidis A, Kontzoglou K, Kouraklis G, Machairas N, Chrysoheris P, Antonakopoulos F, Konstantinidis M, Filippou D, Skandalakis P, Konstantinidis K (2018 Mar-Apr) Short-term outcomes in patients with colon cancer treated with robotic right colectomy. J BUON 23(2):317-321

36. Trastulli S, Desiderio J, Farinacci F, Ricci F, Listorti C, Cirocchi R, Boselli C, Noya G, Parisi A (2013 Jun) Robotic right colectomy for cancer with intracorporeal anastomosis: short-term outcomes from a single institution. Int J Color Dis 28(6):807-814

37. Reitz ACW, Lin E, Rosen SA (2018 Aug) A single surgeon's experience transitioning to robotic-assisted right colectomy with intracorporeal anastomosis. Surg Endosc 32(8):3525-3532

38. Milone M, Elmore U, Allaix ME, Bianchi PP, Biondi A, Boni L, Bracale U, Cassinotti E, Ceccarelli G, Corcione F, Cuccurullo D, Degiuli M, de Manzini N, D’Ugo D, Formisano G, Manigrasso M, Morino M, Palmisano S, Persiani R, Reddavid R, Rondelli F, Velotti N, Rosati R, de Palma GD (2020 Feb) Fashioning enterotomy closure after totally laparoscopic ileocolic anastomosis for right colon cancer: a multicenter experience. Surg Endosc 34(2): $557-563$

39. Cleary RK, Kassir A, Johnson CS, Bastawrous AL, Soliman MK, Marx DS, Giordano L, Reidy TJ, Parra-Davila E, Obias VJ, Carmichael JC, Pollock D, Pigazzi A (2018 Oct 24) Intracorporeal versus extracorporeal anastomosis for minimally invasive right colectomy: a multi-center propensity score-matched comparison of outcomes. PLoS One 13(10):e0206277

40. Liu D, Li J, He P, Tang C, Lei X, Jiang Q, Li T (2019 Mar) Shortand long-term outcomes of totally robotic versus robotic-assisted right hemicolectomy for colon cancer: a retrospective study. Medicine (Baltimore) 98(13):e15028

41. Park JS, Choi GS, Park SY, Kim HJ, Ryuk JP (2012 Sep) Randomized clinical trial of robot-assisted versus standard laparoscopic right colectomy. Br J Surg 99(9):1219-1226

42. Park SY, Choi GS, Park JS, Kim HJ, Choi WH, Ryuk JP (2012 Oct) Robot-assisted right colectomy with lymphadenectomy and intracorporeal anastomosis for colon cancer: technical considerations. Surg Laparosc Endosc Percutan Tech 22(5):e271-e276

43. Trastulli S, Coratti A, Guarino S, Piagnerelli R, Annecchiarico M, Coratti F, di Marino M, Ricci F, Desiderio J, Cirocchi R, Parisi A (2015 Jun) Robotic right colectomy with intracorporeal anastomosis compared with laparoscopic right colectomy with extracorporeal and intracorporeal anastomosis: a retrospective multicentre study. Surg Endosc 29(6):1512-1521

44. D'Annibale A, Pernazza G, Morpurgo E et al (2010 Nov) Robotic right colon resection: evaluation of first 50 consecutive cases for malignant disease. Ann Surg Oncol 17(11):2856-2862

45. Senagore AJ, Delaney CP, Brady KM et al (2004 Nov) Standardized approach to laparoscopic right colectomy: outcomes in 70 consecutive cases. J Am Coll Surg 199(5):675-679

46. Hanna MH, Hwang GS, Phelan MJ, Bui TL, Carmichael JC, Mills SD, Stamos MJ, Pigazzi A (2016) Laparoscopic right hemicolectomy: short- and long-term outcomes of intracorporeal versus extracorporeal anastomosis. Surg Endosc 30(9):3933-3942

47. Milone M, Elmore U, Di Salvo E et al (2015 Aug) Intracorporeal versus extracorporeal anastomosis. Results from a multicentre comparative study on 512 right-sided colorectal cancers. Surg Endosc 29(8):2314-2320

48. Hellan M, Casandra A, Pigazzi A (2009 Jul-Sep) Extracorporeal versus intracorporeal anastomosis for laparoscopic right hemicolectomy. JSLS 13(3):312-317

49. Shapiro R, Keler U, Segev L, Sarna S, Hatib K, Hazzan D (2016 Sep) Laparoscopic right hemicolectomy with intracorporeal 
anastomosis: short-and long-term benefits in comparison with extracorporeal anastomosis. Surg Endosc 30(9):3823-3829

50. Bracale U, Merola G, Cabras F, Andreuccetti J, Corcione F, Pignata G (2018 Jun) The use of barbed suture for intracorporeal mechanical anastomosis during a totally laparoscopic right colectomy: is it safe? A retrospective nonrandomized comparative multicenter study. Surg Innov 25(3):267-273

51. Feroci F, Giani I, Baraghini M, Romoli L, Zalla T, Quattromani R, Cantafio S, Scatizzi M (2018 Dec) Barbed versus traditional suture for enterotomy closure after laparoscopic right colectomy with intracorporeal mechanical anastomosis: a case-control study. Updat Surg 70(4):433-439

Publisher's note Springer Nature remains neutral with regard to jurisdictional claims in published maps and institutional affiliations. 